\title{
Interference Discovery Scheme (IDS) For Cloud Computing
}

\author{
Mr.D.Ragupathi \\ Ph.D., Research Scholar, A.V.V.M Sri Pushpam College (Autonomous), \\ Poondi, Thanjavur, Tamilnadu, India. \\ regupathi_dr13@yahoo.com \\ Dr.N.Jayaveeran \\ Associate Professor, PG \& Research Department of Computer Science, \\ Khadir Mohideen College, Adirampattinam, Tamilnadu, India. \\ njkmc_cs@yahoo.com
}

\begin{abstract}
Giving security in a distributed framework requires more than client confirmation with passwords or advanced authentications and secrecy in information transmission. Distributed model of cloud makes it defenseless and inclined to modern distributed Interference attacks like Distributed Denial of Service (DDOS) and Cross Site Scripting (XSS). To deal with expansive scale arrange get to activity and authoritative control of information and application in cloud, another multi-strung distributed cloud IDS show has been proposed. Our proposed cloud IDS handles extensive stream of information bundles, dissect them and produce reports proficiently by coordinating learning and conduct investigation to distinguish Interferences.
\end{abstract}

Keyword - Cloud Computing; Interference Discovery Scheme; Cloud Security;

\section{INTRODUCTION}

The term cloud is analogical to "Web". The term cloud computing depends on cloud drawings utilized as a part of the past to speak to phone systems and later to delineate web in.

Cloud computing is web based registering where virtual shared servers give programming, framework, stage, gadgets and different assets and facilitating to client as an administration on pay-as you-utilize premise. All the information that a digitized framework brings to the table is given as an administration in the cloud registering model. Clients can get to these administrations accessible on the "web cloud" without having any past knowhow on dealing with the assets included. Cloud clients don't claim the physical framework; rather they lease the utilization from an outsider supplier. They expend assets as an administration and pay just for assets that they utilize. What they just need is a PC and web association. Cloud registering has reformed the IT world with its administrations provisioning framework, less support cost, information and administrations accessibility affirmation, quick availability and versatility. Cloud processing has three essential deliberation layers i.e. framework layer (which is a virtual machine reflection of a server), the stage layer (a virtualized working arrangement of a server) and application layer (that incorporates web applications). Equipment layer is excluded as it doesn't specifically offer to clients. Cloud registering additionally has three administration models to be specific Platform as a Service (PaaS), Infrastructure as a Service (IaaS) and Software as a Service (SaaS) models. PaaS show encourages clients by giving stage on which applications can be produced and run. IaaS convey administrations to clients by keeping up substantial foundations like facilitating servers, overseeing systems and different assets for customers. SaaS show makes client effortless of introducing and running programming administrations all alone machines. By and by, Salesforce.com, Google and Amazon are the main cloud specialist co-ops who augment their administrations for capacity, application and calculation on pay according to utilize premise. Information, application and administrations non-accessibility can be forced through Denial of Service (DOS) or Distributed Denial of Service (DDOS) attacks and both cloud specialist organization and clients get to be disable to give or get cloud administrations. For such kind of attacks Interference Detection System (IDS) can be emplaced as a solid guarded instrument. IDSs are host-based, arrange based and distributed IDSs. Have based IDS (HIDS) screens particular host machines, organize based IDS (NIDS) recognizes Interference s on key system focuses and distributed IDS (DIDS) works both on host and additionally arrange. IDSs deliver cautions for the heads which depend on genuine positives or genuine alerts when really Interference happens and false positive or false alerts if there should arise an occurrence of a wrong discovery by the framework. IDSs can distinguish Interference designs by basically investigating the system bundles, applying marks (pre-characterized governs) and creating alerts for framework chairmen. 
IDS utilizes two strategy for identification i.e. abnormality discovery, that takes a shot at client conduct designs and suspicious conduct. Other technique is abuse location that can recognize through eminent attacks designs and coordinating an arrangement of characterized principles or attacks against framework vulnerabilities through port filtering. Since Cloud framework has colossal system activity, the conventional IDSs are not sufficiently effective to deal with such a huge information stream. Most known IDSs are single strung and because of rich dataset stream, there is a need of multi-strung IDS in Cloud computing condition. In a conventional system, IDS screens, recognizes and caution the authoritative client for system activity by sending IDS on key system stifle focuses on client site. In any case, in Cloud organize IDS must be set at Cloud server site and totally directed and oversaw by the specialist co-op. In this situation, if an aggressor figures out how to infiltrate and harm or take user's information, the cloud client won't be advised straightforwardly. The Interference information would just be imparted through the specialist co-op and client needs to depend on him. The cloud specialist co-op dislike to educate the client about the misfortune and can conceal the data for his picture and notoriety. In such a case, a nonpartisan outsider observing administration can guarantee satisfactory checking and cautioning for cloud client. In this report, we have proposed a proficient multi-strung cloud IDS, controlled and checked by an outsider ID observing administration, who can give ready reports to cloud client and master guidance for cloud specialist organization. With a specific end goal to determine the issues which conventional IDSs cannot resolve, a proficient and solid distributed Cloud IDS model is proposed.

\section{LITERATURE REVIEW}

\section{A. Analysis}

In nowadays a solitary server handles the different solicitations from the client. Here the server needs to prepare the every one of the solicitations from the clients all the while, so the handling time will be high. This may prompts to loss of information and bundles might be postponed and adulterated. On doing this the server can't handle the question from the client in an appropriate way. So the preparing time gets expanded. It might prompts to movement and clog. To defeat these issues we are going for the idea called cloud processing. In this cloud processing we will execute the Proxy server to stay away from these issues. However, in this framework Data Efficiency is enhanced yet not the information security. At whatever point we talk about information effectiveness we ought to talk about information security additionally, in light of the fact that in the cloud processing we don't know from which cloud the information is coming, so in the current framework there is no framework to discover the information security. The framework in view of the new engineering has better adaptability and adaptation to internal failure. A bunch comprises of a solitary server and various intermediary servers and is gotten to by different customers. Intermediary servers stores information on nearby circles and read or compose information determined by a server. The server keeps up the record for all document put away in various intermediaries. At the point when a customer needs to download a few information, it will first send a demand to the Server and the Server then divert the demand to a relating intermediary that have the required information and subsequently the information will be sent to the customer. With the mix of Cloud and Grid processing ideas, the information demand can be productively overhauled in an opportune way. The real piece of the Project is Security, so previously mentioned stage talks about Cloud and Grid Technology, however not about security. The Security execution is accomplished by two stage, in particular Behavioral - Knowledge

Conduct Analysis: Using this strategy, we have to perceive expected conduct (honest to goodness utilize) or an extreme conduct deviation. The system must be effectively prepared to proficiently distinguish Interference s. For a given Interference test set, the system figures out how to recognize the Interference s. Be that as it may, we concentrate on recognizing client behavioral examples and deviations from such examples. With this system, we can cover a more extensive scope of obscure attacks.

Information Analysis: Using a specialist framework, we can portray a noxious conduct with a run the show. One preferred standpoint of utilizing this sort of Interference location is that we can include new standards without altering existing ones. Interference discovery (ID) is a sort of security administration framework for PCs and systems. An ID framework accumulates and examines data from different regions inside a PC or a system to recognize conceivable security ruptures, which incorporate both Interference $s$ (attacks from outside the association) and abuse (attacks from inside the association). ID utilizes weakness evaluation (in some cases alluded to as checking), which is an innovation created to survey the security of a PC framework or system.

Interference identification capacities include:

- Checking and examining both client and framework exercises

- Breaking down framework designs and vulnerabilities

- Surveying framework and record respectability 


\section{B. Existing Technique}

\section{i) Interference discovery for grid and cloud computing}

Cloud and Grid processing are the most defenseless focuses for intruder's attacks because of their distributed condition. For such conditions, Interference Detection System (IDS) can be utilized to upgrade the safety efforts by a deliberate examination of logs, setups and system movement. Customary IDSs are not appropriate for cloud condition as system based IDSs (NIDS) can't identify encoded hub correspondence, additionally have based IDSs (HIDS) are not ready to locate the concealed attacks trail. Eduardo Zied Milian et al. have proposed an IDS benefit at cloud middleware layer, which has a review framework intended to cover attacks that NIDS and HIDS can't recognize. The design of IDS administration incorporates the hub, benefit, occasion inspector and capacity. The hub contains assets that are gotten to through middleware which characterizes get to control arrangements. The administration encourages correspondence through middleware. The occasion reviewer screens and catches the system information, additionally investigates which lead/arrangement is broken. The capacity holds behavior based (correlation of late client activities to regular conduct) and learning based (known trails of past attacks) databases. The reviewed information is sent to IDS benefit center, which dissects the information and alert to be an Interference. The creators have tried their IDS model with the assistance of reproduction and discovered its execution attractive for real-time usage in a cloud domain. Despite the fact that they have not talked about the security strategies consistence check for cloud specialist organization and their detailing techniques to cloud clients.

\section{ii) Interference discovery in the cloud}

Interference identification framework assumes a critical part in the security and tirelessness of dynamic guard framework against gatecrasher threatening attacks for any business and IT association. IDS usage in cloud processing requires a productive, adaptable and virtualization-based approach. In cloud registering, client information and application is facilitated on cloud benefit provider's remote servers and cloud client has a restricted control over its information and assets. In such case, the organization of IDS in cloud turns into the duty of cloud supplier. In spite of the fact that the chairman of cloud IDS ought to be the client and not the supplier of cloud administrations. In the paper, Jeffrey Chan et al. have proposed a mix answer for focal IDS administration that can join and coordinate different prestigious IDS sensors yield gives an account of a solitary interface. The Interference location message trade arrange (IDMEF) standard has been utilized for correspondence between various IDS sensors. The creators have recommended the organization of IDS sensors on partitioned cloud layers like application layer, framework layer and stage layer. Alarms created are sent to „Event Gatherer" program. Occasion gatherer gets and change over ready messages in IDMEF standard and stores in occasion information base vault with the assistance of Sender, Receiver and Handler modules. The investigation part dissects complex attacks and exhibits it to client through IDS administration framework. The creators have proposed a powerful cloud IDS administration design, which could be checked and controlled by the cloud client. They have given a focal IDS administration framework in view of various sensors utilizing IDMEF standard for correspondence and observed by cloud client.

\section{Security Issues in Cloud Computing}

\section{i) Cloud information secrecy issue}

Secrecy of information over cloud is one of the glaring security concerns. Encryption of information should be possible with the conventional procedures. In any case, scrambled information can be secured from a vindictive client yet the protection of information even from the head of information at administration provider's end couldn't be covered up. Seeking and ordering on scrambled information remains a state of worry all things considered. Previously mentioned cloud security issues are a couple and dynamicity of cloud design are confronting new difficulties with quick execution of new administration worldview.

\section{ii) System and host construct attacks with respect to remote Server}

Host and system Interference attacks on remote hypervisors are a noteworthy security worry, as cloud merchants utilize virtual machine innovation. DOS and DDOS attacks are propelled to refuse assistance accessibility to end clients.

\section{iii) Cloud security reviewing}

Cloud reviewing is a troublesome errand to check consistence of all the security strategies by the merchant. Cloud specialist organization has the control of delicate client information and procedures, so a robotized or outsider examining system for information trustworthiness check and legal investigation is required. Protection of information from outsider evaluator is another worry of cloud security 


\section{iv) Absence of information interoperability norms}

It comes about into cloud client information secure state. On the off chance that a cloud client needs to move to other specialist co-op because of specific reasons it would not have the capacity to do as such, as cloud user's information and application may not be good with different vendor's information stockpiling configuration or stage. Security and classification of information would be in the hands of cloud specialist organization and cloud client would be reliant on a solitary specialist organization.

\section{III.PROPOSED MODEL}

\section{A. Work}

Cloud computing gives application and capacity benefits on remote servers. The customers don't need to stress over its upkeep and programming or equipment up-degrees. Cloud show takes a shot at the „concept of virtualization $^{\text {ee }}$ of assets, where a hypervisor server in cloud server farm has various customers on one physical machine. Conveying HIDS in hypervisor or host machine would permit the executive to screen the hypervisor and virtual machines on that hypervisor. However, with the quick stream of high volume of information as in cloud show, there would be issues of execution like over-burdening of VM facilitating IDS and dropping of information parcels. Likewise if host is traded off by a culpable attacks the HIDS utilized on that host would be killed. In such a situation, a system based IDS would be more reasonable for organization in cloud like framework. NIDS would be put outside the VM servers on jug neck of system focuses, for example, switch, switch or door for system activity observing to have a worldwide perspective of the framework. Such NIDS would even now be confronting the issue of expansive measure of information through system get to rate in cloud condition. To deal with an expansive number of information parcels stream in such a domain a multistrung IDS approach has been proposed in this paper. The multi-strung IDS would have the capacity to process expansive measure of information and could decrease the bundle misfortune. After a proficient preparing the proposed IDS would pass the observed alarms to an outsider checking administration, who might thusly specifically illuminate the cloud client about their framework under attacks.

The proposed model is shown in the following figure;

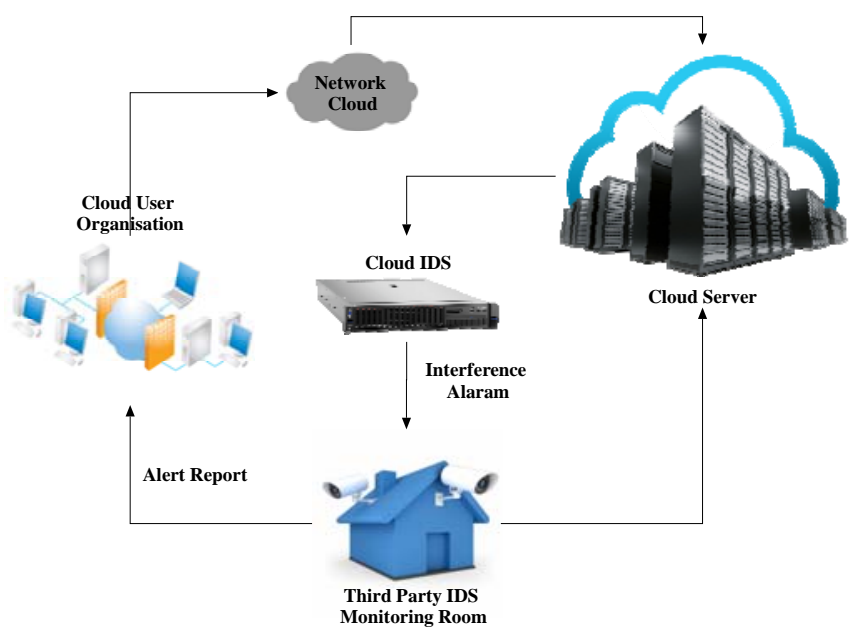

Figure 2. Proposed Cloud IDS Model

The outsider observing administration would likewise give master counsel to cloud specialist co-op for missteps and Interference escape clauses in the framework. Figure 2, demonstrates the proposed IDS show. The cloud client gets to its information on remote servers at administration provider's site over the cloud arrange. Client asks for and activities are observed and logged through a multi-strung NIDS. The ready logs are promptly conveyed to cloud client with a specialist guidance for cloud specialist co-op.

Our proposed multi-strung NIDS demonstrate for distributed cloud condition depends on three modules: catch and lining module, investigation/handling module and detailing module. The catch module, gets the inbound and out-bound (ICMP, TCP, IP, UDP) information parcels. The caught information bundles are sent to the common line for investigation. The examination and process module gets information parcels from the common line and dissect it against mark base and a pre-characterized run set. Each procedure in a common line can have different strings which work in a community oriented model to enhance the framework execution. The principle procedure will get TCP, IP, UDP and ICMP bundles and numerous strings would simultaneously process and match those parcels against pre-characterized set of standards. Through a productive coordinating and examination the awful parcels would be recognized and cautions created. Detailing module would read the alarms from shared line and plans ready reports. The outsider observing and admonitory administration having knowledge and assets would instantly produce a report for cloud user's data and sends a complete master 
counseling report for cloud specialist co-op. Figure 3 portrays the stream diagram of proposed multithreaded Cloud IDS.

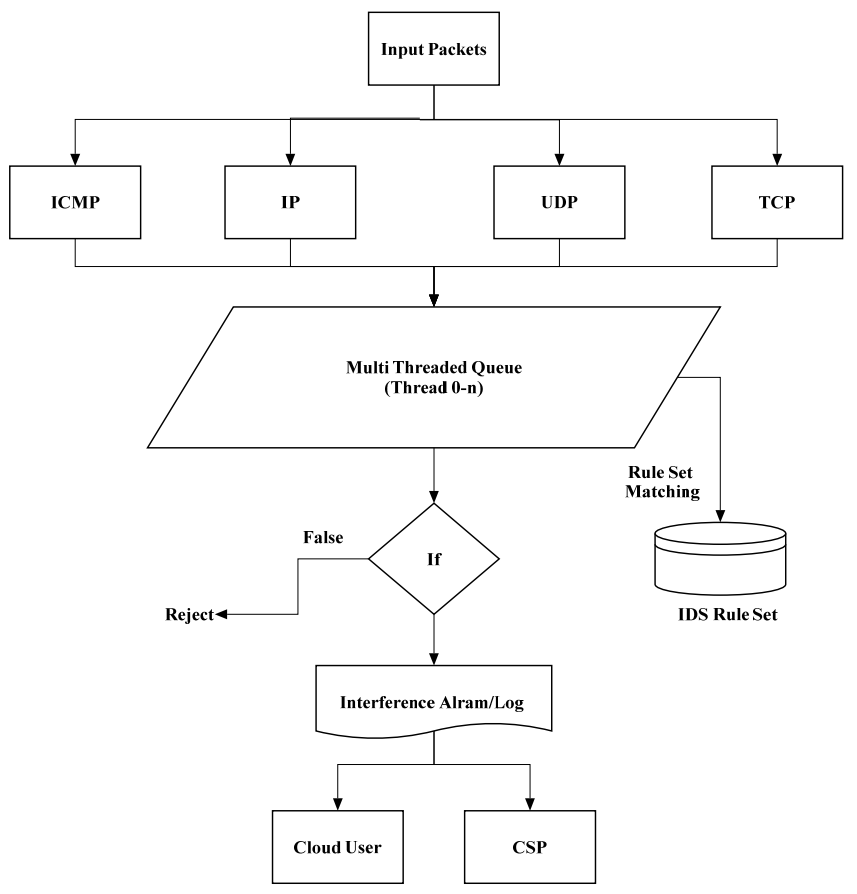

Figure 3. Flow Chart of Multi-Threaded Cloud IDS Model

\section{B. Advantages of proposed model}

- High volume of information in cloud condition could be taken care of by a solitary hub IDS through a multi-strung approach.

- CPU, memory utilization and additionally parcel misfortune would be decreased to enhance the general proficiency of cloud IDS.

- In a host based IDS (HIDS) situation, if have turns into the casualty of culpable assailant and controlled by the gatecrasher, HIDS on that host would be traded off. In such a case the assailant would not permit HIDS to send cautions to director and could play destruction with the information and applications. For better deceivability and resistance, arrange IDS (NIDS) has been proposed for cloud foundation.

- An outsider checking and counseling administration has been proposed, who has both experience and assets to watch/handle Interference information and produce reports for cloud client and additionally consultative reports for cloud specialist organization.

- Being at a main issue, proposed Cloud IDS would be able to complete simultaneous preparing of information examination, which is an effective approach.

\section{IV.CONCLUSION}

Cloud processing is a "system of systems" over the web, accordingly odds of Interference is more with the savviness of intruder's attacks. Diverse IDS strategies are utilized to counter noxious attacks in customary systems. For Cloud computing, huge system get to rate, giving up the control of information and applications to specialist co-op and distributed attacks defenselessness, an effective, dependable and data straightforward IDS is required. In this report, a multi-strung cloud IDS model is proposed which can be directed by an outsider observing administration for a superior upgraded effectiveness and straightforwardness for the cloud client.

\section{REFERENCES}

[1] C.Jen Chung, P.Khatkar, T.Xing, J.Lee \& D.Huang, "NICE: Network Intrusion Detection and Countermeasure Selection in Virtual Network Systems", Dependable and Secure Computing, Volume: 10, Issue: 4, July-Aug. 2013.

[2] Eduardo Zied Milian, Mauro Mesquita Spinola \& Marly Monteiro Carvalho, "Risks and Uncertainties in Cloud Computing: Literature Review, Trends and Gaps", Latin America Transactions, Vol: 15, Issue: 2, Feb. 2017.

[3] G.Somani, M.S.Gaur, D.Sanghi, M.Conti, M.Rajarajan \& R.Buyya, "Combating DDoS Attacks in the Cloud: Requirements, Trends, and Future Directions", in Cloud Computing, Volume: 4, Issue: 1, Jan.-Feb. 2017.

[4] M. H. Ghahramani, MengChu Zhou \& Chi Tin Hon, "Toward cloud computing QoS architecture: analysis of cloud systems and cloud services", Journal of Automatica Sinica, Vol: 4, Issue: 1, Jan. 2017.

[5] Massimo Ficco, Massimiliano Rak, "Stealthy Denial of Service Strategy in Cloud Computing", Cloud Computing, Volume: 3, Issue: 1, March 2015.

[6] Pasquale Donadio, "Virtual intrusion detection systems in the cloud", Bell Labs Technical Journal, Volume: 17, Issue: 3, Dec. 2012. 
[7] Q.Yan, F. Richard Yu, "Distributed denial of service attacks in software-defined networking with cloud computing", in Communications Magazine, Volume: 53, Issue: 4, April 2015.

[8] Suraj R. Pardeshi, Vikul J. Pawar, Kailash D. Kharat, "Enhancing information security in cloud computing environment using cryptographic techniques", Communication and Electronics Systems (ICCES), International Conference on 21-22 Oct. 2016.

[9] Yi Han, Jeffrey Chan, Tansu Alpcan, Christopher Leckie, "Using Virtual Machine Allocation Policies to Defend against Co-Resident Attacks in Cloud Computing", Dependable and Secure Computing, Vol: 14, Issue: 1, Jan.-Feb. 12017.

[10] Yibin Li, Min Chen, Wenyun Dai, Meikang Qiu, "Energy Optimization With Dynamic Task Scheduling Mobile Cloud Computing", IEEE Systems Journal, Volume: 11, Issue: 1, March 2017

[11] Z.Tan, U.T. Nagar, X.He, P.Nanda, R.P.Liu, S.Wang, J.Hu, "Enhancing Big Data Security with Collaborative Intrusion Detection”, in Cloud Computing, Volume: 1, Issue: 3, Sept. 2014. 